\title{
Photophysical Behavior of Coumarins as a Function of Substitution and Solvent: Experimental Evidence for the Existence of a Lowest Lying ${ }^{1}\left(n, \pi^{*}\right)$ State
}

\author{
J. Sérgio Seixas de Melo, ${ }^{\dagger, \ddagger}$ Ralph S. Becker, ${ }^{\dagger}$ and António L. Maçanita ${ }^{*}$, , \\ Centro de Tecnologia Quimica e Biológica, Rua de Quinta Grande, 6, P-2780 Oeiras, Portugal, Departamento \\ de Quimica, Universidade de Coimbra, Coimbra, Portugal, and Departamento de Quimica do IST, \\ Lisboa, Portugal
}

Received: December 14, $1993^{\circ}$

\begin{abstract}
The nature of the lowest excited state for coumarin and some derivatives was investigated using steady-state and time-resolved fluorescence data at room temperature, as well as fluorescence anisotropy at $77 \mathrm{~K}$ in nonpolar and polar solvents in conjunction with theoretical data obtained with different methods: INDO/S-CI, CNDO/ S-CI, MNDO-CI, and AM1-CI. The results show that $S_{1}$ is actually $n, \pi^{*}$ for coumarin in any solvent. Substitution with methoxy and methyl and/or chlorine and/or the increase of solvent polarity reduces the energy gap between the $S_{1}\left(n, \pi^{*}\right)$ and $S_{2}\left(\pi, \pi^{*}\right)$ states, promoting the mixing of these states and finally inducing inversion to $S_{1}$ $\left(\pi, \pi^{*}\right)$ with trisubstitution and for the disubstituted case in dioxane:water (1:4). The presence of a lowest lying $S_{1}\left(n, \pi^{*}\right)$ mediates a large degree of radiationless processes in the singlet mannifold.
\end{abstract}

\section{Introduction}

The assignment of the state order, mixing of states, and their consequence on the photophysics and photochemistry of coumarin systems have been of great interest for some time. ${ }^{1,2}$ This is true not only because of the interest in coumarins but because, in the important psoralens, the coumarin moiety is a central and dominating feature. Both molecular systems constitute important structural classes in their own right but are, in addition, important photosensitizers for both in vitro ${ }^{3}$ and in vivo systems. ${ }^{4}$ In this paper we will be primarily concerned with coumarin itself as well as coumarin with respect to several types of substitution-methyl, methoxy, and chloro, as well as their combination. Hydroxy substitution leads to complex photokinetics, and it was treated separately. 5

There have been a few considerations of primarily lowtemperature (12-77 K) emission spectra of some coumarins which focused mainly on aspects related to phosphorescence. ${ }^{6,7}$ Some fluorescence information including quantum yields is also available for three coumarins 7,8 one of which is in common to those considered here. In all cases, the lowest singlet excited state was assigned as $\pi, \pi^{*}$ which was also the same assignment for the lowest triplet state. For those cases where calculations existed, essentially only coumarin, the assignments were the same.

We will show in this exploration that by careful examination of the fluorescence quantum yields and lifetimes of coumarins as a function of the nature of the solvent and/or substitution, the lowest excited singlet state for coumarin and some derivatives (see Scheme 1) is actually $n, \pi^{*}$ (or essentially $n, \pi^{*}$ ), not $\pi, \pi^{*}$.

Moreover calculations of the INDO/S and CNDO/S type with extensive configuration interaction (CI) (up to 196 configurations for INDO/S and 80 for CNDO/S) also agree with these assignments. We will also show the very considerable dependence of (1) the fluorescence radiative rate constant $k_{\mathrm{F}},(2)$

* Author to whom correspondence should be addressed.

† Rua da Quinta Grande.

¿ Universidade de Coimbra.

Departamento de Química do IST

- Abstract published in Advance ACS Abstracts, May 15, 1994.

\section{SCHEME 1}<smiles></smiles>

$$
\begin{aligned}
& \text { (C) } R_{1}=R_{2}=R_{3}=H \\
& \text { (CIC) } R_{1}=R_{3}=H ; R_{2}=C l \\
& (M M C) R_{1}=M e ; R_{2}=H ; R_{3}=O M e \\
& (C I M M C) R_{1}=M e ; R_{2}=C l ; R_{3}=O M e
\end{aligned}
$$

the sum of the nonradiative rate constants $\left(k_{\mathrm{nr}}\right)$, and (3) the state order upon the nature of the solvent and, in some cases, upon the nature and degree of substitution. Some laser transient data for 6-methylcoumarin is also considered.

\section{Experimental Section}

Coumarin $(\mathrm{C})$ and 3-chlorocoumarin $(\mathrm{ClC})$ were purchased from Aldrich and Kodak and used without further purification. 7-Methoxy-4-methylcoumarin (MMC) was synthesized by methylation of 7-hydroxy-4-methyl-coumarin with dimethyl sulfoxide in aqueous media following standard procedures.9 The compound was purified by successive recrystallization from ethanol/water mixtures. 3-Chloro-7-methoxy-4-methylcoumarin (CIMMC) was synthesized from its hydroxy analogue 3-chloro7-hydroxy-4-methylcoumarin ${ }^{10}$ following the same procedure as described above for MMC. Purification of ClMMC was performed by column chromatography. With the four compounds, the absence of fluorescent impurities was assured by the quality of single-exponential fits of the fluorescence decays. Water was twice distilled and passed through a Millipore (Millipore Milli-Q grade) apparatus. Ethanol (Merck, Uvasol) was dried over $\mathrm{CaO}$ and than passed through a silica column. Methylcyclohexane (Riedel de Häen for Synthesis) was purified as elsewhere reported. ${ }^{11}$ All the other solvents (spectroscopic grade) 
TABLE 1: Absorption Spectral Data in Cyclohexane (Wavelength, $\lambda$ and Extinction Coefficients, $\epsilon$ ) and Theoretical Predictions (Wavelength, $\lambda$, and Oscillator Strength, $f$ for Coumarin (C), 3-Chlorocoumarin (CIC). 7-Methoxy-4-methylcoumarin (MMC), and 3-Chloro-7-methoxy-4-methylcoumarin (CIMMC)

\begin{tabular}{|c|c|c|c|c|c|c|c|c|}
\hline \multirow[b]{2}{*}{ compd } & \multicolumn{2}{|c|}{ exptla } & \multicolumn{2}{|c|}{$\mathrm{CNDO} / \mathrm{S}-\mathrm{CI}^{a}$} & \multicolumn{2}{|c|}{ INDO/S-CI ${ }^{a}$} & \multirow{2}{*}{$\begin{array}{c}\text { MNDO-CI } \\
\lambda(\mathrm{nm})\end{array}$} & \multirow{2}{*}{$\begin{array}{c}\text { AM1-CI } \\
\lambda(\mathrm{nm})\end{array}$} \\
\hline & $\lambda(\mathrm{nm})$ & $\epsilon\left(\mathrm{M}^{-1} \mathrm{~cm}^{-1}\right)$ & $\overline{\lambda(\mathrm{nm})}$ & $f$ & $\overline{\lambda(\mathrm{nm})}$ & $f$ & & \\
\hline \multirow[t]{3}{*}{ C } & & & 332 & $0.0004 n, \pi^{*}$ & 347 & $0.0007 n, \pi^{*}$ & 335 & \\
\hline & 312 & 5700 & 308 & 0.08 & 297 & 0.12 & 314 & 309 \\
\hline & 272 & 11400 & 271 & 0.11 & 271 & 0.47 & & 292 \\
\hline \multirow[t]{3}{*}{$\mathrm{CIC}$} & & & & & 347 & $0.0005 n, \pi^{*}$ & 340 & \\
\hline & 314 & 7200 & & & 312 & 0.24 & 321 & 316 \\
\hline & 282 & 12300 & & & 282 & 0.26 & & 298 \\
\hline \multirow[t]{2}{*}{$\mathrm{MMC}^{b}$} & & & 324 & $0.001 n, \pi^{*}$ & & & 337 & \\
\hline & $\begin{array}{l}318 \\
287\end{array}$ & $\begin{array}{r}13000 \\
8700\end{array}$ & $\begin{array}{l}317 \\
286\end{array}$ & $\begin{array}{l}0.1 \\
0.15\end{array}$ & & & 324 & $\begin{array}{l}314 \\
298\end{array}$ \\
\hline \multirow[t]{2}{*}{$\mathrm{CIMMC}^{e}$} & & & & & 335 & $0.005 n, \pi^{*}$ & 346 & \\
\hline & $\begin{array}{l}326 \\
295\end{array}$ & $\begin{array}{l}9600 \\
5650\end{array}$ & & & $\begin{array}{l}332 \\
295\end{array}$ & $\begin{array}{l}0.350 \\
0.190\end{array}$ & 326 & $\begin{array}{l}324 \\
301\end{array}$ \\
\hline
\end{tabular}

a All transition are $\pi \rightarrow \pi^{*}$ unless otherwise noted. ${ }^{b}$ Other experimental transitions probably exist in the $240-250$ - and 200-210-nm regions. c The INDO/S calculation used 7-hydroxy instead of 7-methoxy. However, on the basis of other calculations, the differences should only be 1-2 nm. Also, another experimental transition probably exists in the $\approx 290$-nm region.

were used without further purification, and none of them showed residual fluorescence at the wavelengths of interest. The solutions $\left(1 \times 10^{-5} \mathrm{M}\right.$ ) were deoxygenated by $\mathrm{N}_{2}$ or Ar bubbling.

Absorption and fluorescence spectra were run with a Beckman DU-70 and a SPEX Fluorolog spectrometers, respectively. The fluorescence spectra were corrected for the wavelength response of the system. The fluorescence quantum yields were measured using several standards, namely, methyl 1-pyrenoate $\left(\phi_{\mathrm{F}}=0.83\right.$ in cyclohexane $\left.{ }^{11}\right)$ and methyl 1-naphthoate $\left(\phi_{F}=0.48_{g}\right.$ in cyclohexane ${ }^{11}$ ).

Fluorescence decays were obtained using the time-correlated single-photon-timing technique, as previously described, ${ }^{12}$ except for the wavelength shift, which is now $300 \mathrm{fs} / \mathrm{nm}$. The time resolution of this apparatus is 100 ps. $^{5}$ The fluorescence decays were deconvoluted in a MicroVax 3100 computer, employing the method of modulating functions. ${ }^{14}$

Molecular orbital calculations were performed in a MicroVax 2000 or MicroVax 3100 using several methods: CNDO/S-CI, INDO/S-CI, MNDO-CI, and AM1-CI. The CNDO/S-CI method $^{13}$ (QCPE, Department of Chemistry, Indiana University) was used for $C$ and $M M C$, while calculations of the intermediate neglect of differential overlap type (INDO) were performed for $\mathrm{C}, \mathrm{ClC}$, and CIMMC with the INDO/S-CI model. ${ }^{15}$

Atomic coordinates for the INDO/S calculations were obtained from minimum energy geometries determined by a PC model molecular modeling software program (Serena software, Bloomington, IN) employing an MMX force field (including $\pi$ system routines). For the other calculations, the initial coordinates were calculated using the DTMM program ${ }^{16}$ and then optimized using the AMPAC software package ${ }^{17}$ QCPE.

The results obtained with the "spectroscopy" programs were compared with those from MNDO-CI and AM1-CI. The twocenter electron repulsion integrals for INDO/S were estimated using the Mataga-Nishimoto approximation. ${ }^{18}$

\section{Results}

Figure 1 shows the absorption spectra of $(\mathrm{C})$ and its derivatives, 3-chlorocoumarin (ClC), 7-methoxy-4-methylcoumarin (MMC), and 3-chloro-7-methoxy-4-methylcoumarin (CIMMC) in cyclohexane. Their structural formula and designations as we are going to consider them here are depicted in Scheme 1. Substitution with a chlorine or methoxy group affects the two near-UV bands in different ways; namely, the methoxy group strongly affects the spectral shape, while the chlorine atom only induces a red shift. Both bands, which are clearly of $\pi \rightarrow \pi^{*}$ nature (see below, $\epsilon$ values in Table 1), are red-shifted in the order $\mathrm{ClC} \approx \mathrm{MMC}$ and ClMMC.

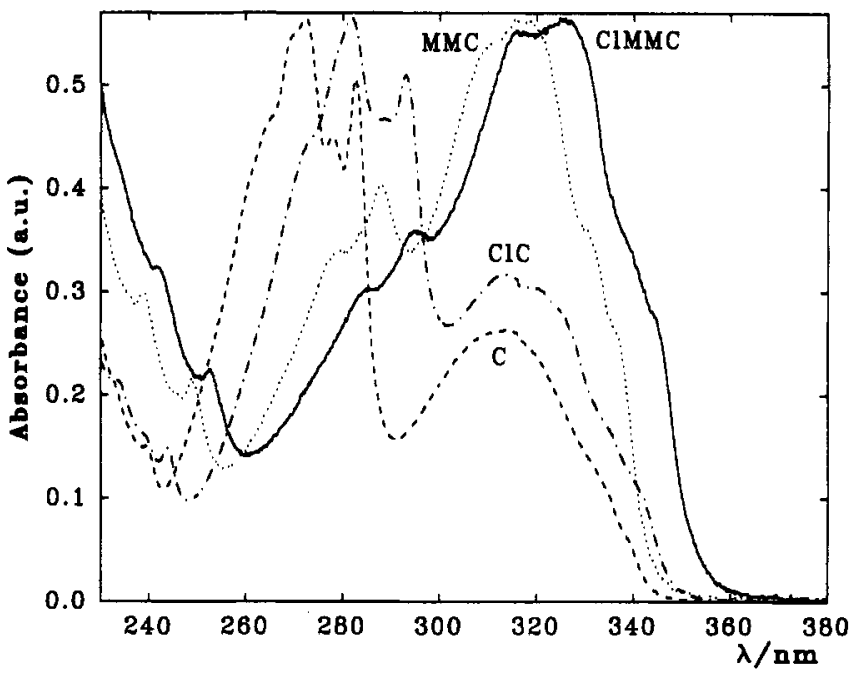

Figure 1. Absorption spectra of (C) coumarin (- -), (CIC) 3-chlorocoumarin (-*-), (MMC) 7-methoxy-4-methylcoumarin (*..), and ClMMC) 3-chloro-7-methoxy-4-methylcoumarin (-) in cyclohexane at 20 ${ }^{\circ} \mathrm{C}$.

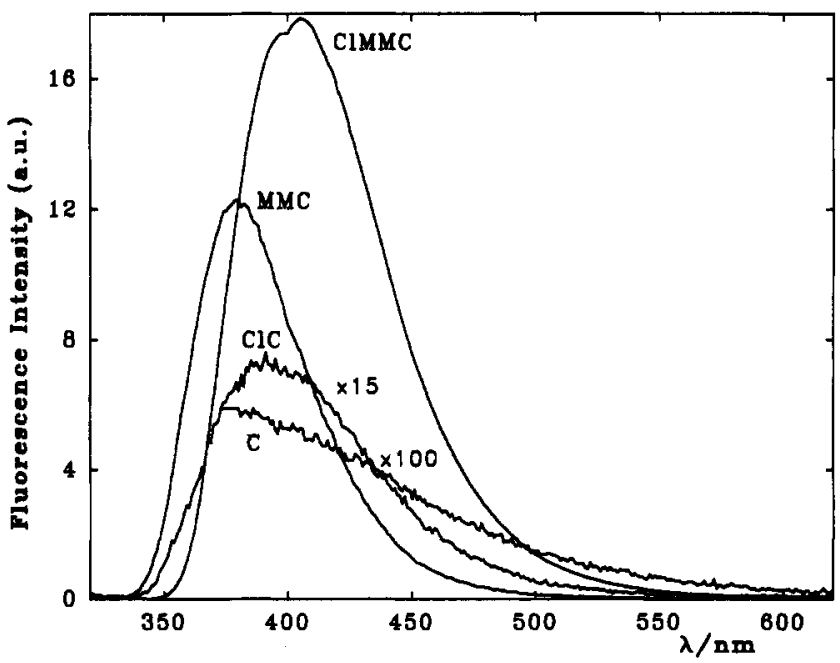

Figure 2. Fluorescence emission spectra of coumarin (C), 3-chlorocoumarin (ClC), 7-methoxy-4-methylcoumarin (MMC), and 3-chloro-7methoxy-4-methylcoumarin (CIMMC) in a dioxane:water mixture of 1:4. Spectra of $(\mathrm{C})$ and $(\mathrm{ClC})$ are 100-and 15-fold magnified, respectively.

Table 1 presents absorption spectral data as well as comparative theoretical data for the coumarins concern.

The fluorescence intensity of coumarins strongly depends on substitution (note that spectra of $\mathrm{C}$ and $\mathrm{ClC}$ in Figure 2 are 100 - 
TABLE 2: Experimental Values of the Fluorescence Quantum Yields $\left(\phi_{F}\right)$, Lifetimes $\left(\tau_{F}\right)$, and Radiative $\left(\boldsymbol{k}_{\mathrm{r}}\right)$ and Radiationless $\left(k_{\text {ur }}\right)$ Rate Constants in Cyclohexane $(C x)$, Dioxane $(D x)$, and a Dioxane:Water Mixture of 1:4 $\left(D x: H_{2} 0\right.$, 1:4) of Coumarin $(C)$, 3-Chlorocoumarin (CIC), 7-Methoxy-4-methylcoumarin (MMC), and 3-Chloro-7-methoxy-4-methylcoumarin (CIMMC)

\begin{tabular}{|c|c|c|c|c|c|c|}
\hline compd & solvent & $\phi_{\mathrm{F}}$ & $\tau_{\mathrm{F}}(\mathrm{ns})$ & $k_{\mathrm{F}}\left(\mathrm{ns}^{-1}\right)$ & $k_{\mathrm{nr}}\left(\mathrm{ns}^{-1}\right)$ & $\tau_{\mathrm{F}}^{0 a}(\mathrm{~ns})$ \\
\hline \multirow[t]{3}{*}{ C } & $\mathrm{Cx}$ & 0.0003 & $\leq 0.10$ & $\geq 0.003$ & $\geq 10.0$ & $\leq 330$ \\
\hline & $\mathrm{Dx}$ & 0.0004 & $\leq 0.10$ & $\geq 0.004$ & $\geq 10.0$ & $\leq 250$ \\
\hline & Dx: $\mathrm{H}_{2} \mathrm{O}, 1: 4$ & 0.0020 & 0.10 & 0.02 & 10.0 & 50 \\
\hline \multirow[t]{3}{*}{$\mathrm{CIC}$} & $\mathrm{Cx}$ & 0.0004 & $\leq 0.10$ & $\geq 0.004$ & 10.0 & $\leq 250$ \\
\hline & Dx & $0.002_{6}$ & 0.10 & 0.026 & 10.0 & 39 \\
\hline & $\mathrm{Dx}: \mathrm{H}_{2} \mathrm{O}, 1: 4$ & 0.0220 & 0.31 & 0.071 & 3.2 & 14 \\
\hline \multirow[t]{3}{*}{ MMC } & $\mathrm{Cx}$ & 0.0014 & 0.10 & 0.014 & 10.0 & 71 \\
\hline & Dx & 0.014 & 0.13 & 0.11 & 7.6 & 9.1 \\
\hline & Dx: $\mathrm{H}_{2} \mathrm{O}, 1: 4$ & 0.62 & 1.70 & 0.36 & 0.22 & 2.8 \\
\hline \multirow[t]{3}{*}{ CIMMC } & $\mathrm{Cx}$ & 0.12 & 0.64 & 0.19 & 1.40 & 5.3 \\
\hline & Dx & 0.51 & 2.40 & 0.21 & 0.19 & 4.8 \\
\hline & $\mathrm{Dx}: \mathrm{H}_{2} \mathrm{O}, 1: 4$ & 0.83 & 3.90 & 0.21 & 0.06 & 4.8 \\
\hline
\end{tabular}

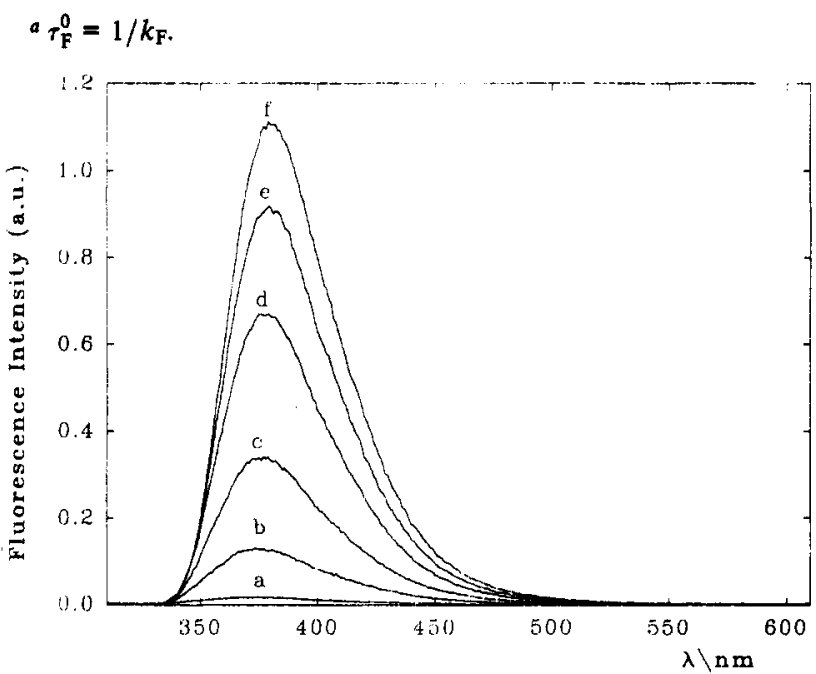

Figure 3. Fluorescence emission spectra $\left(\lambda_{e x}=337 \mathrm{~nm}\right)$ of 7 -methoxy4-methylcoumarin (MMC) at $20^{\circ} \mathrm{C}$ in (a) dioxane; (b) dioxane:water, $4: 1$; (c) dioxane:water, 3:2; (d) dioxane:water, 2:3; (e) dioxane:water, $1: 4$; and (f) water.

and 15-fold magnified, respectively) and on solvent polarity (Figure 3), where the fluorescence spectra of $\mathrm{MMC}$ in $\mathrm{Dx}: \mathrm{H}_{2} \mathrm{O}$ mixtures are shown as an example.

Table 2 presents data for $\mathrm{C}, \mathrm{ClC}, \mathrm{MMC}$, and ClMMC regarding the quantum yield of fluorescence $\left(\phi_{\mathrm{F}}\right)$ and lifetime of fluorescence $\left(\tau_{F}\right)$ in nonpolar (cyclohexane), slightly polar (dioxane), and polar (1:4 dioxane:water $\left(\mathrm{Dx}: \mathrm{H}_{2} \mathrm{O}\right)$ mixture) solvents. The 1:4 dioxane:water mixture was chosen because of its high dielectric constant and hydrogen bonding ability, which makes it much more polar than most ordinary solvents. ${ }^{19,20}$ Pure water was not used in order to avoid precipitation and microaggregation phenomena.

In Table 3, data for $\phi_{\mathrm{F}}, \tau_{\mathrm{F}}, k_{\mathrm{r}}$, and $k_{\mathrm{nr}}$ of $\mathrm{MMC}$ as a function of the Dimroth polarity parameter $\left(E_{\mathrm{T}}(30)\right)$ is presented over a still broader range of solents and solvent mixtures. The fluorescence quantum yields in a number of nonpolar, polar aprotic, and protic solvents and solvent mixtures show that $\phi_{\mathrm{F}}$ increases 2 orders of magnitude on going from cyclohexane to water. This response to solvent polarity is also reflected on the fluorescence lifetime of MMC.

Fluorescence anisotropy of MMC and CIMMC was measured in methylcyclohexane and ethanol at $77 \mathrm{~K}$ as well as in a viscous nonpolar solvent (USP Oil; $\eta^{20}{ }^{\circ} \mathrm{C} \approx 60 \mathrm{cP}$ ) at room temperature. The $r$ values ${ }^{21}$ are approximately constant within the wavelength range of interest $(230-380 \mathrm{~nm})$ and are as follows: $r(\mathrm{MMC} /$ $\mathrm{MCH})=-0.09, r(\mathrm{MMC} / \mathrm{EtOH})=0.3$ at $77 \mathrm{~K}$, and $r(\mathrm{ClMMC} /$ USP) $=0.11$ at $20^{\circ} \mathrm{C}$. This last solvent was chosen due to its high viscosity value, which reduces random reorientation of the molecules.
TABLE 3: Fluorescence Ouantum Yields $\left(\phi_{F}\right)$, Lifetimes $\left(\tau_{\mathrm{F}}\right)$, and Radiative $\left(k_{\mathrm{F}}\right)$ and Radiationless $\left(k_{\mathrm{m}}\right)$ Rate Constants of 7-Methoxy-4-methylcoumarin (MMC) in n-Hexane (n-Hx), Cyclohexane (Cx), Dioxane (Dx), Dichloromethane $\left(\mathrm{CH}_{2} \mathrm{Cl}_{2}\right)$, Ethanol (EtOH), Methanol (MeOH), Water $\left(\mathrm{H}_{2} \mathrm{O}\right)$, and Dioxane:Water $\left(\mathrm{Dx}_{\mathbf{H}} \mathrm{H}_{2} \mathrm{O}\right)$ Mixtures as a Function of the $E_{\mathrm{T}}(\mathbf{3 0})$ Parametere

\begin{tabular}{|c|c|c|c|c|c|}
\hline solvent & $\begin{array}{c}E_{\mathrm{T}}(30) \\
\left(\mathrm{kcal}-\mathrm{mol}^{-1}\right)\end{array}$ & $\phi_{F}$ & $\tau_{\mathrm{F}}(\mathrm{ns})$ & $k_{\mathrm{F}}\left(\mathrm{ns}^{-1}\right)$ & $k_{\mathrm{nr}}\left(n \mathrm{~s}^{-1}\right)$ \\
\hline$n-\mathrm{Hx}$ & 30.9 & 0.0014 & 0.10 & 0.014 & 10.20 \\
\hline$C x$ & 31.2 & 0.0014 & 0.10 & 0.014 & 10.20 \\
\hline Dx & 36.0 & 0.0140 & 0.13 & 0.110 & 7.60 \\
\hline $\mathrm{CH}_{2} \mathrm{Cl}_{2}$ & 41.1 & 0.0370 & 0.13 & 0.280 & 7.30 \\
\hline $\mathrm{Dx}: \mathrm{H}_{2} \mathrm{O}, 4: 1$ & 49.0 & 0.0980 & 0.33 & 0.300 & 2.76 \\
\hline $\mathrm{Dx}: \mathrm{H}_{2} \mathrm{O}, 3: 2$ & 51.6 & 0.2480 & 0.67 & 0.370 & 1.12 \\
\hline EtOH & 51.9 & 0.1800 & 0.57 & 0.320 & 1.44 \\
\hline $\mathrm{Dx}: \mathrm{H}_{2} \mathrm{O}, 2: 3$ & 53.6 & 0.4760 & 1.18 & 0.400 & 0.44 \\
\hline $\mathrm{MeOH}$ & 55.5 & 0.1240 & 0.35 & 0.360 & 2.48 \\
\hline $\mathrm{Dx}: \mathrm{H}_{2} \mathrm{O}, 1: 4$ & 57.0 & 0.6200 & 1.70 & 0.370 & 0.22 \\
\hline $\mathrm{H}_{2} \mathrm{O}$ & 63.1 & 0.6980 & 1.90 & 0.370 & 0.16 \\
\hline
\end{tabular}

${ }^{a} E_{\mathrm{T}}(30)$ values of $\mathrm{Dx}: \mathrm{H}_{2} \mathrm{O}$ mixtures are from ref 24 .

\section{Discussion}

Our first consideration will be the assignment of the states order. Several pieces of data are important regarding this aspect, but we shall begin with an examination of coumarin. Note, in Table 2, that for coumarin the fluorescence radiative lifetime ( $\tau_{\mathrm{F}}^{0}=1 / k_{\mathrm{F}}$ ) in cyclohexane or dioxane is greater than $250 \mathrm{~ns}$. Clearly this long lifetime is appropriate for an emission originating from a singlet state of quite highly forbidden character. On the basis of the integrated absorption of the first clear observable transition with a maximum at $313 \mathrm{~nm}$ (Figure 1), the radiative lifetime would be predicted to be 4-8 ns (eq 122 ). Clearly, this

$$
\frac{1}{\tau_{0}}=\frac{8 p(2303) c n^{2}}{N\langle\bar{\nu}\rangle_{\mathrm{av}}^{-1}} \frac{g_{1}}{g_{2}} \int \epsilon \mathrm{d} \ln \bar{\nu}
$$

latter is some 50-fold shorter than actually observed, and therefore, this lowest observable $\pi \rightarrow \pi^{*}$ transition is not the state from which emission occurs. Even in a $1: 4$ dioxane: $\mathrm{H}_{2} \mathrm{O}$ mixture $\tau_{\mathrm{F}}^{0}$ is some 10 times longer than predicted by eq 1 , indicating emission from a forbidden-like state.

Four coumarin, our calculations predict the $n \rightarrow \pi^{*}$ transition to be lowest. Our general experience in comparing calculated and observable $n \rightarrow \pi^{*}$ transitions indicates that if the theory predicts an $\left(n, \pi^{*}\right)$ state approximately $\geq 2000 \mathrm{~cm}^{-1}$ below a $\left(\pi, \pi^{*}\right)$ state, then it is likely that the predicted ordering is correct. In the case of coumarin, the values of $2300 \mathrm{~cm}^{-1}$ (CNDO/S) and $4850 \mathrm{~cm}^{-1}$ (INDO/S) for such a separation would provide strong probability that the $\left(n, \pi^{*}\right)$ is lowest. Of course, the experimental radiative fluorescence lifetime is compatible with the emission originating from a state having essentially ${ }^{1}\left(n, \pi^{*}\right)$ character. 
For the 3-chlorocoumarin, the radiative lifetime in cyclohexane is $\approx 250 \mathrm{~ns}$, (Table 2). Again, it is clear that this lifetime is compatible only with emission from a quite highly forbidden state. Parallel to the case of coumarin, on the basis of the integrated area of the first clearly observable transition, a lifetime of 4-8 ns would be expected if emission occurred from this $\pi, \pi^{*}$ state. Thus, the lowest emitting state must be principally of $n, \pi^{*}$ character as for coumarin. Note that the calculations also clearly indicate that the $n \pi^{*}$ singlet state is the lowest of the singlets. However, in a dioxane:water mixture of $1: 4$, the radiative lifetime has shortened by a large factor ( $\approx 18$ fold) to $14 \mathrm{~ns}$. Although this is note quite as short as that predicted from the integrated absorption, considering potential errors in the integrated absorption and calculations, it is close. In any event it is clear that considerable $\pi, \pi^{*}$ character has been mixed into the lowest $n, \pi^{*}$ state, and the lowest excited singlet state could be considered principally $\pi, \pi^{*}$ in character.

In the case of 7-methoxy-4-methylcoumarin (MMC) in cyclohexane, the radiative lifetime is $71 \mathrm{~ns}$ (from $\phi_{\mathrm{F}}$ and $\tau_{\mathrm{F}}$ ), Table 2. On the basis of an estimate of the radiative lifetime from the absorption band (Figure 1), $\tau_{F}^{0}$ would be about 2-6 ns. Again note that this latter lifetime is $12-35$-fold shorter than measured, indicating that the emitting state has significant forbidden character and, therefore, does not originate from the lowest observed $\pi \rightarrow \pi^{*}$ transition/state (with maximum $\approx 318$ $\mathrm{nm})$. Theoretical calculations indicate an $\mathrm{n}, \pi^{*}$ state as the lowest, although now, the lowest $n, \pi^{*}$ and $\pi, \pi^{*}$ states are much closer together than in the case of coumarin (see Table 1). In fact they are close enough that theory alone could not be utilized to predict the correct order. The considerably shortened lifetime is consistent with a lowest lying ${ }^{1}\left(n, \pi^{*}\right)$ vibronically mixed/coupled state to a relatively close lying upper allowed ${ }^{1}\left(\pi, \pi^{*}\right)$ state. However the mixing of $\pi, \pi^{*}$ character into the $n, \pi^{*}$ state still does not appear to be so dominant that that state could be called simply $\pi, \pi^{*}$.

If the solvent is changed to methanol, water, or a dioxane: water mixture of $1: 4$, then the radiative lifetime of MMC undergoes a dramatic shortening to $\approx 3 \mathrm{~ns}$ (from $\approx 70 \mathrm{~ns}$ in cyclohexane; see Tables 2 and 3 ). The $\approx 3$-ns lifetime is well within the range predicted for fluorescence from a lowest allowed ${ }^{1}\left(\pi, \pi^{*}\right)$ state (see discussion above). Therefore, the above solvents have caused an inversion of the two lowest excited states such that now the ${ }^{1}\left(\pi, \pi^{*}\right)$ state is the lowest.

In the case of 3-chloro-7-methoxy-4-methylcoumarin (CIM$\mathrm{MC}$ ), the $\phi_{\mathrm{F}}$ in dioxane is 0.51 and the measured lifetime is 2.4 ns. In this case the radiative lifetime is $4.8 \mathrm{~ns}$. On the basis of the integrated absorption of the first observable band of the $\pi \rightarrow$ $\pi^{*}$ type (see Figure 1), the radiative lifetime would be expected to be 2-6 ns, similar to that of MMC. Note the very close agreement to that measured. Theoretical calculations indicate that the ${ }^{1}\left(n, \pi^{*}\right)$ state is still fairly low, but the difference is extremely small $\left(\approx 250 \mathrm{~cm}^{-1}\right)$ compared with coumarin (Table 1 ), and again, theory alone certainly cannot be trusted to accurately predict the correct state order. Clearly the very close agreement between the calculated predicted radiative lifetime and that measured (based on $\phi_{F}$ and $\tau_{F}$ ) strongly indicates that the lowest singlet excited state is of the ${ }^{1}\left(\pi, \pi^{*}\right)$ nature. This is in distinct contrast to coumarin and 3-chlorocoumarin, as well as still different from MMC.

To summarize briefly at this stage. The evidence is very strong that, in coumarin, a basically pure ${ }^{1}\left(n, \pi^{*}\right)$ state is the lowest state, and on the basis of the radiative lifetime, a ${ }^{1}\left(\pi, \pi^{*}\right)$ state is above, and "considerably" above, the ${ }^{1}\left(n, \pi^{*}\right)$ state. On the basis of a value of $\epsilon \approx 120 \mathrm{~mol} \mathrm{dm}^{-3} \mathrm{~cm}^{-1}$, the estimated radiative lifetime of the ${ }^{1}\left(n, \pi^{*}\right)$ state would be in the 200-400-ns range. This is in fact what is observed, indicating relatively little mixing of the upper ${ }^{1}\left(\pi, \pi^{*}\right)$ state into the lowest ${ }^{1}\left(n, \pi^{*}\right)$ state, and therefore, they are "considerably" separated. Theoretical cal- culations also indicate a fairly substantial separation (INDO/S or $\mathrm{CNDO} / \mathrm{S}$ ).

Substitution of chlorine at the 3 position $(\mathrm{ClC})$ has no effect on the state order present in coumarin itself. Moreover, on the basis of the radiative lifetime in cyclohexane, there is a little effect of mixing the ${ }^{1}\left(\pi, \pi^{*}\right)$ state into the lowest ${ }^{1}\left(n, \pi^{*}\right)$ state. Nevertheless, the $n, \pi^{*}-\pi, \pi^{*}$ energy gap becomes smaller than that for $C$, as seen from the 2 order of magnitude increase of $\phi_{F}$ on going from cyclohexane to $\mathrm{Dx}: \mathrm{H}_{2} \mathrm{O}(1: 4)$ mixture (and the large decrease in $\tau_{\mathrm{F}}^{0}$ ).

Substitutions of methyl in position 4 and methoxy in position 7 (MMC) do induce a change in the mixing of the ${ }^{1}\left(\pi, \pi^{*}\right)$ and ${ }^{1}\left(n, \pi^{*}\right)$ states. On the basis of the radiative lifetime (measured), the ${ }^{1}\left(n, \pi^{*}\right)$ state appears to still be lowest in cyclohexane but now the ${ }^{1}\left(\pi, \pi^{*}\right)$ state is considerably closer and mixes to cause a shortening of the radiative lifetime compared to coumarin. In the (1:4) $\mathrm{Dx}: \mathrm{H}_{2} \mathrm{O}$ solvent mixture, that state order is inverted and the $\pi, \pi^{*}$ state is consequently now the lowest.

Finally, for the trisubstituted case ClMMC, the chlorine induces a significant red shift of the $\pi \rightarrow \pi^{*}$ transition relative to MMC and INDO/S calculations predict practically isoenergetic ${ }^{1}\left(n, \pi^{*}\right)$ and $1\left(\pi, \pi^{*}\right)$ states. Now, the radiative lifetime is totally commensurate with the fluorescence originating from an essentially pure ${ }^{1}\left(\pi, \pi^{*}\right)$ state both in cyclohexane and $\mathrm{Dx}: \mathrm{H}_{2} \mathrm{O}$ $(1: 4)$. Then obviously, this is now the lowest state and the ${ }^{1}\left(\mathrm{n}, \pi^{*}\right)$ is now higher.

The above conclusions are consistent with the fluorescence anisotropy results, which indicate that the emitting state of MMC depends on the solvent polarity. In the nonpolar solvent (MCH), the anisotropy of MMC is negative and close to zero, indicating that the absorption and the fluorescence transition dipole moments are not aligned. Since the lowest two $\pi, \pi^{*}$ transition dipole moments lie roughly in the same direction (according to our MO calculations), we must conclude that the emitting state is very probably $n, \pi^{*}$. On the contrary, the anisotropy value of MMC in ethanol $(r=0.3)$ at $77 \mathrm{~K}$ indicates that both transition dipoles are basically in the same direction; ${ }^{21}$ i.e., the $\pi, \pi^{*}$ state is lowest. In the case of ClMMC a positive value for $r(r=0.11)$ is obtained even at room temperature in a nonpolar viscous solvent; i.e., the $\pi, \pi^{*}$ state is lowest even for a nonpolar solvent.

Our results for coumarin are in disagreement with those of others, ${ }^{6-8,23}$ where the ${ }^{1}\left(\pi, \pi^{*}\right)$ state was assigned as lowest. Still others later made a similar assignment based on the origin of a phosphorescence excitation spectrum relative to the origin of fluorescence ${ }^{6}$ or by rationalizing a low quantum yield of intersystem crossing from $T_{2} \rightarrow S_{1}$ (and the relative location for $\mathrm{T}_{1} \pi, \pi^{*}$ state).$^{8}$ The earlier assignments ${ }^{7,23}$ were based primarily on low-temperature data $(77 \mathrm{~K})$ in ethanol and some theoretical calculations. Of the theoretical methods used, ${ }^{7,23}$ the CNDO/S method would be capable of predicting $n, \pi^{*}$ transitions but it appears that no $\mathrm{CI}$ was involved. In such a case the reliability of predicting the relative location of the $n, \pi^{*}$ transition would be very poor (in fact it was predicted to be $1 \mathrm{eV}$ above the lowest $\left.\pi, \pi^{*}\right)$. On the basis of positive polarization data $(77 \mathrm{~K}$, in ethanol), they ${ }^{7,23}$ assigned the lowest excited singlet state as $\left(\pi, \pi^{*}\right)$. In dry isopentane at $77 \mathrm{~K}$ it was found that the fluorescence polarization was significantly less positive. The reason for the depolarization was not known, but the lowest excited singlet state was still assigned as $\pi, \pi^{*}$.

Our data in Dx: $\mathrm{H}_{2} \mathrm{O}(1: 4)$, which has a higher $E_{\mathrm{T}}(30)$ than ethanol, still indicate a ${ }^{1}\left(n, \pi^{*}\right)$ lowest state. However, it could be at $77 \mathrm{~K}$ in ethanol that an upper ${ }^{1}\left(\pi, \pi^{*}\right)$ state mixes sufficiently with a lowest ${ }^{1}\left(n, \pi^{*}\right)$ state so that one would observe a weak fluorescence with induced positive polarization, but, even then, a ${ }^{1}\left(n, \pi^{*}\right)$-like state would be lowest.

On the basis of our data, experiment, and theory and that of others, we assign the relative and absolute energies of several excited singlet and triplet states of $C$, as in Figure 4. Others ${ }^{6-8,23}$ 
$S_{s}, x^{*}: 46000+2000 \mathrm{~cm}^{-1}$

$\mathrm{S}_{4} \frac{\pi \pi^{*}: 44600 \pm 1000 \mathrm{~cm}^{-1}}{\pi, \pi^{*}: 4600 \pm 2000 \mathrm{ct}}$

$S_{3} \underline{x, x^{*}: 36800+400 \mathrm{~cm}^{-1}}$

$S_{2}, x, x^{*}: 32800 \pm 400 \mathrm{~cm}^{-1}$

$S_{1} \stackrel{n, \pi^{*}: 29100 \pm 500 \mathrm{~cm}^{-1}}{ }$

$$
T_{5} \frac{\pi, \pi^{*}: 38000+2000 \mathrm{~cm}^{-1}}{T_{4} \pi, \pi^{*}: 35000+2000 \mathrm{~cm}^{-1}}
$$

$\mathrm{T}_{3}, \mathrm{n}, \pi^{*}: 28000 \pm 300 \mathrm{~cm}^{-1}$

$T_{2} \frac{\pi . \pi^{*}: 26000 \pm 1000 \mathrm{~cm}^{-1}}{T^{2}}$

$T_{1} \pi, \pi^{*}: 22000 \pm 300 \mathrm{~cm}^{-1}$

\section{$\mathrm{S}_{0}$}

Figure 4. Energies of the singlet and triplet states of coumarin based on maxima except for $T$.

have the order $T_{\pi, \pi^{*}}<T_{n, \pi^{*}}<S_{\pi, \pi^{*}}<S_{n, \pi^{*}:}$ note we have two $T_{\pi, \pi^{*}}$ states (as well as a $T_{n, \pi^{*}}$ state) below a lowest $S_{n, \pi^{*}}$.

Note that, given the $\phi_{F}$ and $\tau_{F}$ data, more than $98 \%$ of the quanta are lost through radiationless processes to the ground state although in which mannifold, $S$ or $\mathrm{T}$, this is dominant cannot be determined from such data. However, for 6-methylcoumarin, the $\phi_{\mathrm{T}}$ is only 0.04 (benzene $298 \mathrm{~K}$ ) 25 and the $\phi_{\mathrm{F}}$ would appear to be in the range of $0.01-0.02$ (ethanol, $77 \mathrm{~K}$ ). ${ }^{23}$ Also for coumarin and 5,7-dimethoxycoumarin in water low values of $\phi_{\mathrm{T}}$ were found, 0.054 and 0.072 , respectively. ${ }^{26}$ Thus, it is very likely that the greatest majority of the quanta are lost within the singlet mannifold for both 6-methylcoumarin and coumarin itself. It is clearly of note that changing the solvent to more polar ones (or higher $E_{\mathrm{T}}(30)$ ) does increase $\phi_{\mathrm{F}}$ but still it remains very low $(<0.01)$. This pattern closely resembles the one found with 5 -methoxypsoralen, for which the internal conversion rate constant decreases more than 1 order of magnitude from dioxane to a dioxane:water $(1: 4)$ mixture, ${ }^{20}$ also due to the proximity of the two lowest $n, \pi^{*}$ and $\pi, \pi^{*}$ singlet excited states. ${ }^{27}$ It is also of salient interest that certain multisubstitutions include chloro, methyl, and methoxy groups, do dramatically increase $\phi_{\mathrm{F}}$ (to 0.83 for CIMMC from 0.002 for $C$ in the same solvent; Table 2) and thus drastically reduce radiationless processes: note that it is clear that a lowest state order change has occurred from $n, \pi^{*}$ to $\pi, \pi^{*}$. This happens for lesser substitution as well but only in a highly polar $\left(E_{\mathrm{T}}(30)\right)$ solvent (mixture). It is then quite apparent that a lowest ${ }^{1}\left(n, \pi^{*}\right)$ state mediates significant radiationless processes (in the singlet mannifold).

\section{Conclusions}

The fluorescence properties of coumarin and its derivatives strongly depend on the energy difference or order of the two close-lying lowest singlet states ( $\mathrm{n} \pi^{*}$ and $\pi \pi^{*}$ ). For coumarin, $S_{1}\left(n \pi^{*}\right)$ is well below $S_{2}\left(\pi \pi^{*}\right)$ and the solvent-induced decrease of the energy difference ( $\pi \pi^{*}$ red shift and $n \pi^{*}$ blue shift) is not sufficient to allow significant $\mathrm{n} \pi^{*}-\pi \pi^{*}$ mixing.

However, for substituted coumarins, the $\pi \pi^{*}-n \pi^{*}$ energy differences become smaller with increasing substitution, and the states order may be inverted. For the intermediate cases, where $S_{1}\left(n \pi^{*}\right)$ lies only slightly bellow $S_{2}\left(\pi \pi^{*}\right)$, the nature of $S_{1}$ is modulated by the solvent, leading to changes of $k_{\mathrm{F}}$ larger than 1 order of magnitude on going from nonpolar to polar solvents.

The internal conversion process $\left(k_{\text {ic }}\right)$ seems also be strongly dependent on the $S_{1}$ nature (at least in the case of coumarin); i.e., $k_{\mathrm{ic}}$ increases with the $\mathrm{n} \pi^{*}$ contribution on $\mathrm{S}_{1}$. Such phenomena have been clearly demonstrated for 5-methoxypsoralen, ${ }^{20}$ on the basis of $\phi_{\mathrm{T}}$ data in a large number of solvents.

Acknowledgment. A.L.M. thanks the Alexander von Humbolt Foundation, Drs. K. Zacchariasse and R. Busse for their support for the construction of the single-photon-counting equipment, and G. Striker for making his deconvolution programs available. This work was supported by JNICT (Grant No. STRD/C/AMB/ 40/92). J.S.M. also acknowledges JNICT for a Ph.D. Grant (No. BD/839/90-IF).

\section{References and Notes}

(1) Haydon, S. C. Spectrosc. Lett. 1975, 8, 815.

(2) Schulman, S. G. In Molecular Luminescence Spectroscopy; Schulman, S., Ed.; Wiley-Interscience: New York, 1985; Vol. 1.

(3) Giese, A. C. Photophysiology 1971, 6, 114.

(4) Song, P. S.; Harter, M. L.; Moore, T. A.; Herndon, W. C. Photochem. Photobiol. 1971, 14, 521

(5) Seixas de Melo, J.; Maçanita, A. L. Chem. Phys. Lett. 1993, 204, 556.

(6) Harrigan, E. T.; Chakrabarti, A.; Hirota, N. J. Am. Chem. Soc. 1976, 98,3460

(7) Mantulin, W. W.; Song, P. S. J. Am. Chem. Soc. 1973, 95, 5122. $188,49$.

(8) Chou, P. T.; Martinez, M. L.; Studer, S. L. Chem. Phys. Lett. 1992,

(9) Voguel, A. I. A textbook of practical organic chemistry including qualitative organic analysis; Longmans and Green: London, 1966.

(10) Chakravarti, D. J. Indian Chem. Soc. 1931, 8, 129.

(11) Maçanita, A. L.; Magalhães, J.; Dias, A.; Teles, H.; Iglésias, E. J. Chem. Soc., Faraday Trans. 1990, 86, 4011.

(12) Maçanita, A. L.; Costa, F. P.; Costa, S. M.; Melo, E. C.; Santos, H. J. Phys. Chem. 1989, 93, 336.

(13) Quantum Chemistry Program Exchange Program No. QCMP034, Department of Chemistry, Indiana University, Bloomington, IN.

(14) Striker, G. In Effective Implementation of Modulation Functions in Deconvolution and Reconvolution of Analytical Signals; Bouchy, M., Ed.; University Press: Nancy, France, 1982.

(15) Ridley, J. E.; Zerner, M. C. Theor. Chim. Acta 1973, 32, 111.

(16) Desktop Molecular Modeller; John Appleyard, Polyhedron Software, and M. James C. Crabbe, University of Reading; Oxford University Reading Press: Oxford, U.K., 1989.

(17) Dewar, M. J. S.; Bingham, R. C.; Lo, D. H. J. Am. Chem. Soc. 1975, 97,1285 .

(18) Nishimoto, K.; Mataga, N. Z. Phys. Chem. (Frankfurt) 1957, 12, 335 .

(19) Melo, E. C.; Costa, S. M. B.; Maçanita, A. L.; Santos, H. J. Colloid Interface Sci. 1991, 141, 439.

(20) Melo, T. S.; Maçanita, A. L.; Prieto, M.; Bazin, M.; Ronfard-Haret, J. C.: Santus, R. Photochem. Photobiol. 1988, 47, 429.

(21) Lakowicz, J. R. Principles of Fluorescence Spectroscopy; Plenum Press: New York, 1983.

(22) Becker, R. S. Theory and Interpretation of Fluorescence and Phosphorescence; Wiley-Interscience: New York, 1969.

(23) Song, P. S.; Gordon, W. H. J. Phys. Chem. 1970, 74, 4234.

(24) Langhals, H. Angew. Chem., Int. Ed. Engl. 1982, 22, 711.

(25) Becker, R. S.; Chakravorti, S.; Gartner, C. A.; Miguel, M. G. J. Chem. Soc., Faraday Trans. 1993, 89, 1001.

(26) Land, E. J.; Truscott, T. G. Photochem. Photobiol. 1979, 29, 861.

(27) Lai, T.-L.; Lim, B. T.; Lim, E. C. J. Am. Chem. Soc. 1982, 104, 7631. 